\title{
In Vitro and In Vivo Activities of Macrolides against Mycobacterium leprae
}

\author{
SCOTT G. FRANZBLAU* AND ROBERT C. HASTINGS \\ Gillis W. Long Hansen's Disease Center, Carville, Louisiana 70721
}

Received 20 June 1988/Accepted 8 September 1988

\begin{abstract}
We previously demonstrated the potent in vitro activity of erythromycin against Mycobacterium leprae as determined by its effect on ATP pools and rates of palmitate oxidation and phenolic glycolipid I synthesis. In the present study, the relative in vitro activities of a number of new macrolides with superior pharmacokinetic properties were assessed. In addition, for the most active compounds, concentrations in serum were determined by bioassay during continuous administration in the feed of mice, and in vivo activity against $M$. leprae was assessed by the kinetic mouse footpad technique. Both clarithromycin and roxithromycin were more potent than erythromycin in vitro, with the former showing the highest activity in accelerating rates of ATP decay and reducing rates of palmitate oxidation. In mice, concentrations of clarithromycin in serum were higher than those of roxithromycin and erythromycin, with the latter undetectable even when administered at $0.1 \%$ (wt/wt) in the diet. When administered at $0.01 \%(\mathrm{wt} / \mathrm{wt})$ in the diet, erythromycin and roxithromycin were unable to inhibit growth of $M$. leprae in mouse footpads whereas clarithromycin demonstrated bactericidal-type activity. On the basis of these data and other properties of macrolides, a clinical trial of clarithromycin in leprosy is warranted.
\end{abstract}

The existing armamentarium of antileprosy drugs is limited in number, and only one, rifampin, is rapidly bactericidal. In addition, drug-resistant strains of Mycobacterium leprae have been reported to the most commonly used agents (15). These factors among others have led to an ongoing search for new antileprosy agents.

Because of the inability to cultivate the leprosy bacillus, primary drug screening has relied on the use of the mouse footpad system (MFP), which has, because of cost, time requirement, need for relatively large amounts of test drug, and necessity for favorable pharmacokinetic properties in mice, limited the number of drugs that can be evaluated. Therefore, a number of in vitro systems have been developed which rely on the inhibition of metabolic activity as an indicator of drug activity $(8,10,11,13,14,17,19,25)$. We have demonstrated the potent direct activity of erythromycin against $M$. leprae as determined by measurement of intracellular ATP (11), radiorespirometric assay of palmitate oxidation (8), and measurement of the rate of phenolic glycolipid I synthesis (E. B. Harris, S. G. Franzblau, and R. C. Hastings, Int. J. Lepr., in press). We hypothesized that the failure of erythromycin to inhibit growth in the MFP (R. Gelber, personal communication) may be due to poor pharmacokinetics when the drug is administered in the feed of mice. Recently, a number of semisynthetic macrolides which have superior acid stability and serum half-lives compared with erythromycin have been developed $(2,3,6$, $7,24)$. In this paper, we assess the in vitro and in vivo activities of some of these compounds against the leprosy bacillus.

\section{MATERIALS AND METHODS}

Inoculum preparation. $M$. leprae suspensions were prepared from the footpads of infected HSD nu/nu mice (Harlan Sprague-Dawley, Indianapolis, Ind.) as previously described (8). Briefly, footpads were surface decontaminated by using UV irradiation, ethyl ether, $1 \%$ iodine, and $70 \%$ ethanol.

\footnotetext{
* Corresponding author.
}

Footpads were then minced and manually homogenized in Dubos broth base without polysorbate 80 (pH 6.5) (GIBCO Diagnostics, Madison, Wis.) and containing $20 \%$ (vol/vol) Dubos medium albumin (Difco Laboratories, Detroit, Mich.), $50 \mu \mathrm{g}$ of ampicillin per $\mathrm{ml}$, and $2.5 \mu \mathrm{g}$ of amphotericin B per $\mathrm{ml}$. These antibiotics have shown no effect on $M$. leprae in vitro or in vivo. Complete medium is referred to as Dubos medium albumin (DA). For experiments assessing the effect of macrolides by subsequent measurement of palmitic acid oxidation, bacilli were partially purified by slow-speed centrifugation $\left(108 \times g, 5 \mathrm{~min}, 10^{\circ} \mathrm{C}\right)$ to remove tissue debris, high-speed centrifugation of the supernatant $(2,710 \times g, 45$ min, $10^{\circ} \mathrm{C}$ ) to pellet bacilli, and resuspension of the pellet in $10 \mathrm{ml}$ of DA. The suspension was held at $4^{\circ} \mathrm{C}$ for 1 day and then at $33^{\circ} \mathrm{C}$ for 1 day. Subsamples inoculated into a variety of culture media confirmed a lack of contaminating bacteria. For experiments assessing the effects of macrolides in vitro by ATP analysis or in vivo by growth in mouse footpads, bacilli were more rigorously purified as previously described $(8,23)$. Briefly, the homogenate was treated with collagenase and DNase at $33^{\circ} \mathrm{C}$ for $1 \mathrm{~h}$ with intermittent mild agitation. Bacilli were first partially purified by differential centrifugation as described above and then layered over two-step discontinuous Percoll gradients consisting of a cushion of 2 $\mathrm{ml}$ of $100 \%$ Percoll and $8 \mathrm{ml}$ of $50 \%$ Percoll in 1:5-diluted Dulbecco phosphate-buffered saline without calcium chloride and sodium bicarbonate (Sigma Chemical Co., St. Louis, Mo.). Gradients were centrifuged at 2,710 $\times g$ for 1.5 $h$ at $15^{\circ} \mathrm{C}$. The lower bands were removed from the interface and diluted with DA, and the bacilli were pelleted at $2,710 \times$ $g$ for $30 \mathrm{~min}$ at $15^{\circ} \mathrm{C}$, washed, and resuspended in DA. Cell counts were determined microscopically as described by Shepard and McRae (21).

Drugs. Dapsone (4,4'-diaminodiphenyl sulfone), rifampin, erythromycin base, and erythromycin ethylsuccinate were obtained from Sigma. Azithromycin (CP-62, 993; XZ-450; Pfizer Central Research, Groton, Conn.), clarithromycin (A-56268; TE-031; Abbott Laboratories, North Chicago, Ill.), roxithromycin (RU 965; RU 28965; Hoechst-Roussel 
Pharmaceuticals Inc., Somerville, N.J.), and M-119-31 and M-119-49 (Kirin Brewery Co., Ltd., Tokyo, Japan) were gifts from the manufacturers. For in vitro studies, all compounds were solubilized in absolute ethanol at 1 to $2 \mathrm{mg} / \mathrm{ml}$ and immediately filter sterilized and diluted in DA to $100 \times$ final desired concentrations. Then $10 \mu \mathrm{l}$ was added to $1-\mathrm{ml}$ bacterial cultures. For pharmacokinetic and in vivo antimicrobial assessments, the macrolides were mixed in mouse chow with a twin-shell blender (Patterson-Kelley Co., East Stroudsburg, Pa.).

In vitro drug susceptibility. (i) Radiorespirometry. $M$. leprae were diluted in DA to a density of $10^{7} / \mathrm{ml}$, and $1-\mathrm{ml}$ portions were distributed to 6-ml glass screw-cap vials as previously described (8). After addition of antimicrobial agents, vials were incubated at $33^{\circ} \mathrm{C}$ with loose caps in chambers which were flushed on days 1,4 , and 6 with $2.5 \%$ $\mathrm{O}_{2}-10 \% \mathrm{CO}_{2}-87.5 \% \mathrm{~N}_{2}$ (Scott Medical Products, Plumsteadville, Pa.) for $5 \mathrm{~min}$ at $10 \mathrm{lb} / \mathrm{in}^{2}$. On day $7,1 \mu \mathrm{Ci}$ of $\left[1-{ }^{14} \mathrm{C}\right]$ palmitic acid $(58 \mathrm{mCi} / \mathrm{mmol}$; Dupont, NEN Research Products, Boston, Mass.) was added to each vial in $10 \mu \mathrm{l}$ of ethanol. The glass vials with loose caps were placed in wide-mouth plastic scintillation vials containing a hollow cylinder of filter paper which had previously been saturated with a concentrated liquid scintillation fluor and alkalinized with $100 \mu l$ of $1 \mathrm{~N} \mathrm{NaOH}$. The entire assembly was then incubated at $33^{\circ} \mathrm{C}$, and the evolved ${ }^{14} \mathrm{CO}_{2}$ was quantitated at $1,3,5$, and 7 days by placing the entire assembly in a model LS-5801 liquid scintillation counter (Beckman Instruments, Inc., Fullerton, Calif.).

(ii) ATP assay. Percoll-purified cells of $M$. leprae were diluted in DA to a density of $3.1 \times 10^{7} / \mathrm{ml}$, and 1 -ml portions were distributed to $1.5-\mathrm{ml}$ screw-cap microfuge tubes as described previously (11). After overnight incubation at $33^{\circ} \mathrm{C}$, antimicrobial agents were added and the culture tubes, with loose caps, were incubated for 3 weeks in an airtight chamber which was constantly flushed with air via an aquarium pump. Bacilli were washed two times in TrisEDTA buffer and solubilized by boiling in the presence of chloroform, and then ATP was determined by the firefly bioluminescence assay as previously described (11).

Determination of macrolides in mouse serum. Macrolides were administered to BALB/c mice in their feed at concentrations of 0.01 and $0.1 \%$ (wt/wt) for 3 days. Four mice from each group were then bled by cardiac puncture at 6-h intervals for $24 \mathrm{~h}$. Drug concentrations in serum were determined by bioassay, using Micrococcus luteus ATCC 9341. An 8-ml portion of a 48-h tryptic soy broth culture was diluted into $330 \mathrm{ml}$ of molten $\left(50^{\circ} \mathrm{C}\right)$ antibiotic medium 11 (Difco), and 12-ml portions were dispensed into petri dishes (grid, 150 by $20 \mathrm{~mm}$; medium depth, $2 \mathrm{~mm}$ ). Then $20 \mu \mathrm{l}$ of serum or drug standards (diluted in normal mouse serum) were applied to 740-E disks (Schliecher \& Schuell, Inc., Keene, Notto), which were placed on the agar surface. Cultures were incubated overnight at $30^{\circ} \mathrm{C}$. Drug concentrations in serum were determined from standard curves of the respective macrolides which had been generated by plotting the log standard concentration versus the square of the diameter of the zone of inhibition.

In vivo drug susceptibility. A slight modification of the kinetic method of Shepard (20) was used to assess drug activity against $M$. leprae in mouse footpads. A 33- $\mu$ l portion containing $5 \times 10^{3}$ Percoll-purified, nude-mouse-derived $M$. leprae was inoculated into both hind footpads of $60 \mathrm{BALB} /$ c mice. All mice received normal chow for 30 days, after which groups of 10 mice received chow containing $0.01 \%$ macrolide for 60 days while control mice continued to receive normal chow. All mice were then placed on normal chow for the remainder of the experiment. Three control mice were sacrificed at appropriate time intervals, and the extent of multiplication of $M$. leprae was assessed by microscopic observation of acid-fast bacilli in six individual footpad homogenates as described by Shepard (20). When six of six individual footpads (three mice) were positive $(\geq 1.6 \times$ $10^{4}$ acid-fast bacilli per footpad), three mice (six footpads) from each drug-treated group were also evaluated in an identical manner. The process was repeated at selected intervals to determine the duration of inhibition.

\section{RESULTS}

In vitro activity. Consistent with previous results (8), in vitro exposure of $M$. leprae to erythromycin at $\geq 2 \mu \mathrm{g} / \mathrm{ml}$ for 1 week effected a significant reduction in subsequent oxidation of $\left[1-{ }^{14} \mathrm{C}\right]$ palmitic acid (Fig. 1). Macrolide M-119-49 had similar activity, whereas M-119-31 and roxithromycin appeared somewhat more active. Azithromycin demonstrated the weakest activity of all the macrolides, whereas clarithromycin was the most active compound, causing significant inhibition at $0.125 \mu \mathrm{g} / \mathrm{ml}$. Similar results were found by measuring bacillary ATP concentrations after 3 weeks of exposure to these macrolides (Fig. 2). Although all of the macrolides caused lower ATP concentrations than in controls, the relative activities of erythromycin, roxithromycin, and clarithromycin were the same as described above.

Concentrations in serum. There was no detectable antimicrobial activity in the sera of mice receiving erythromycin ethylsuccinate at either 0.01 or $0.1 \%$ (wt/wt) (Table 1 ). Roxithromycin could be detected only in the sera of mice receiving the higher drug concentration. Clarithromycin was detected in mice receiving $0.01 \%$ (wt/wt) drug but was not maintained above $156 \mathrm{ng} / \mathrm{ml}$ throughout the 24-h testing period. When clarithromycin was administered at $0.1 \%$ (wt/ wt), relatively high concentrations were detected in mouse sera.

In vivo activity. Percoll-purified $M$. leprae from nude mice, growing in control BALB/c mice, reached plateau phase at 3 to 4 months postinoculation (Fig. 3). Growth in erythromycin-treated mice was not significantly different from growth in controls throughout the course of the experiment. In roxithromycin-treated mice, four of six footpads were positive at 4 months postinoculation and all were positive by 5 months postinoculation. In contrast, $M$. leprae did not reach detectable levels $\left(1.6 \times 10^{4}\right)$ in mice receiving clarithromycin even 5 months after discontinuation of drug treatment $(8$ months postinoculation), which indicated bactericidal activity.

\section{DISCUSSION}

The MFP, while the only widely accepted technique for determining the viability of $M$. leprae, suffers from a number of shortcomings as a preliminary drug-screening system for leprosy. In addition to requiring gram quantities of new compounds for determination of maximum tolerated dose, and requiring 6 to 12 months to complete, the system relies on favorable pharmacokinetics in a nonhuman system. The potent in vitro activity of erythromycin against $M$. leprae suggests that the failure of this compound in vivo is due to an inability to achieve adequate concentrations in serum (and thus tissue) when administered in the feed of infected mice. In humans, the acid lability of erythromycin can be minimized by use of an enteric coating. Although the short 


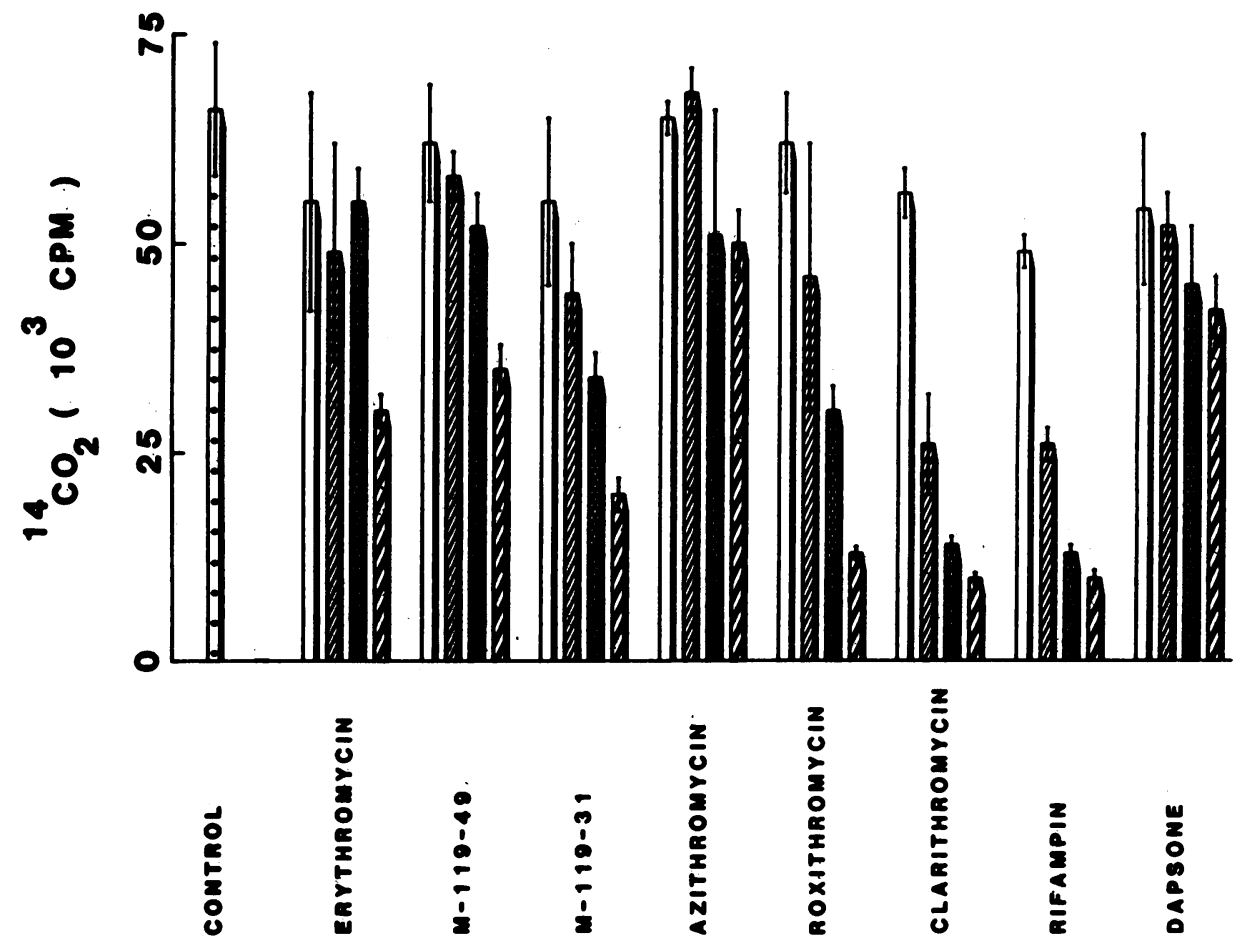

FIG. 1. In vitro effects of macrolides on palmitate oxidation by $M$. leprae. Bacilli $\left(10^{7}\right)$ were incubated with $0(H), 0.031(||), 0.125(h)$, 0.5 ( ), or $2(\boldsymbol{h}) \mu \mathrm{g}$ of drug per $\mathrm{ml}$ for 1 week. $\left[1-{ }^{14} \mathrm{C}\right]$ palmitic acid $(1 \mu \mathrm{Ci})$ was then added, and evolved ${ }^{14} \mathrm{CO}_{2}$ was assayed after $1 \mathrm{week}$. Bars represent means \pm standard deviations of quadruplicate samples except for the control group (16 replicates).

half-life of erythromycin in serum would preclude its use in treating leprosy (because of the need for daily multiple dosing), its potent in vitro activity led us to pursue the investigation of new macrolides with improved pharmacokinetic properties.

Erythromycin has not been used as an antimycobacterial agent, although there are at least a couple of reports of its activity against mycobacteria $(1,18)$. Clarithromycin had previously been shown to be active in vitro against Mycobacterium kansasii, Mycobacterium xenopi, and Mycobacterium fortuitum but inactive against Mycobacterium avium

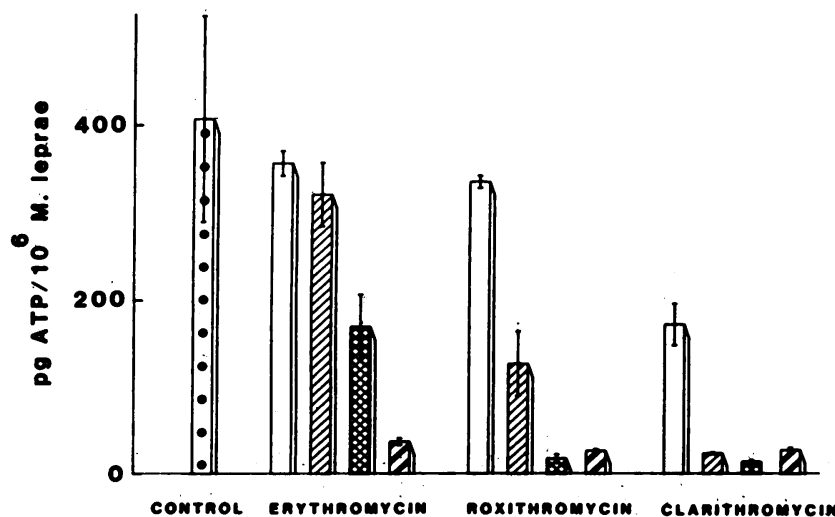

FIG. 2. In vitro effects of macrolides on intracellular ATP pools in $M$. leprae. Percoll-purified bacilli $\left(3.1 \times 10^{7}\right)$ were incubated with

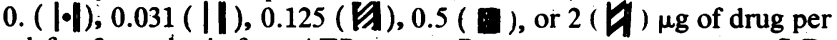
$\mathrm{ml}$ for 3 weeks before ATP assay. Bars represent means \pm S.D. standard deviations of quadruplicate samples except for the control group (13 replicates). and Mycobacterium tuberculosis (3). To our knowledge, this is the first report of the in vivo activity of clarithromycin against any mycobacterium. The pharmacokinetics of roxithromycin are reportedly superior to those of erythromycin, although not sufficiently so to make the drug active in the MFP at $0.01 \%$ (wt/wt). If higher drug concentrations can be achieved in humans, this compound may have potential in treating clinical leprosy. Azithromycin was developed to have an expanded spectrum of activity, and indeed it has activity against some gram-negative organisms (2) as well as excellent pharmacokinetics. However, azithromycin was considerably less active in vitro than were the other macrolides against gram-positive cocci (2), results that may be consistent with the relatively poor activity of azithromycin against $M$. leprae in vitro.

The kinetic MFP technique was developed to differentiate bacteristatic and bactericidal antileprosy drugs. We slightly modified this technique by using a nude-mouse-derived, Percoll-purified $M$. leprae inoculum which we have found to be enriched for metabolically active bacilli (23). This modi-

TABLE 1. Levels of macrolides in sera of BALB/c mice ${ }^{a}$

\begin{tabular}{lcc}
\hline \multicolumn{1}{c}{ Macrolide } & \multicolumn{2}{c}{$\begin{array}{c}\text { Range of concn }(\mu \mathrm{g} / \mathrm{ml}) \text { over } 24 \mathrm{~h} \\
\text { during administration in diet } \\
\text { at }(\%[\mathrm{wt} / \mathrm{wt}]):\end{array}$} \\
\cline { 2 - 3 } & 0.01 & 0.1 \\
\hline $\begin{array}{l}\text { Erythromycin ethylsuccinate } \\
\text { Roxithromycin }\end{array}$ & $<0.156$ & $<0.156$ \\
Clarithromycin & $<0.156$ & $0.219-0.350$ \\
\hline
\end{tabular}

${ }^{a}$ Mice received macrolides in feed for 3 days and were then bled at 6-h intervals for $24 \mathrm{~h}$. Concentrations in serum were determined by bioassay, using Micrococcus luteus. 


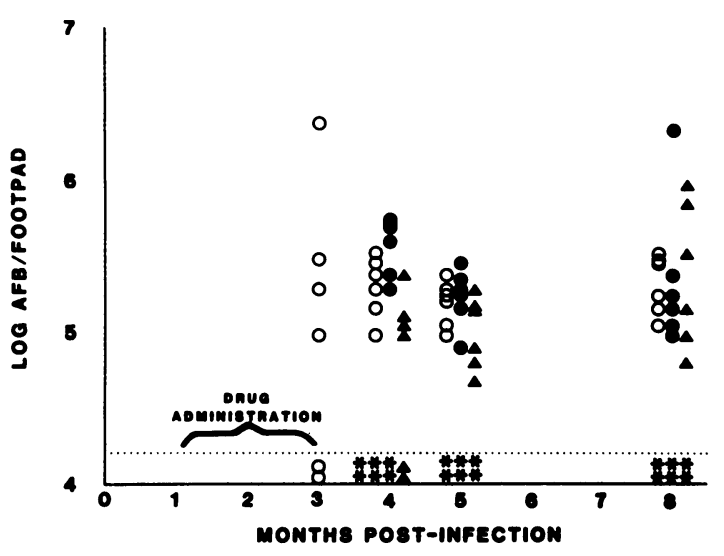

FIG. 3. Effects of macrolides on growth of $M$. leprae in mice. A total of $5 \times 10^{3}$ nude-mouse-derived, Percoll-purified $M$. leprae were inoculated into both hind footpads of BALB/c mice. Data points represent individual footpads of mice receiving chow containing no drug $(\bigcirc)$ or $0.01 \%(w t / w t)$ erythromycin ethylsuccinate $(\bullet)$, roxithromycin $(\Delta)$, or clarithromycin (*) from days 30 through 90 postinfection, after which all mice received drug-free chow. $M$. leprae were enumerated by direct microscopic observation. Dotted line represents threshold of detectability.

fication apparently had the desired effect of shortening the time required to reach plateau phase, and thus we administered drugs from days 30 through 90 postinoculation instead of the traditional days 60 through 120 used with inocula in which this would correspond to the mid-logarithmic phase of growth. When administered at $0.01 \%$ (wt/wt) in the diet, at least one drug (clarithromycin) had a peak concentration in serum exceeding that effecting an in vitro reduction in palmitate oxidation and ATP concentration. Therefore, this concentration, which corresponds to about $100 \mathrm{mg} /$ day in humans, was selected for in vivo testing. Compounds that delay growth of $M$. leprae for a period of time equal to or less than the period of drug administration are considered bacteristatic, whereas those causing a growth delay longer than the period of growth are considered bactericidal. Relatively few compounds, including rifampin, clofazimine, dapsone (weak), thioamides, thiacetazone, some fluoroquinolones, and certain cephalosporins (22), have shown bactericidal activity against $M$. leprae in the MFP. An effective therapeutic regimen for leprosy should include at least one bactericidal drug if that drug is to be used intermittently, e.g., once monthly. Thus, the bactericidal activity of clarithromycin in the present study is of considerable interest.

Although it is tempting to try to quantitatively correlate in vitro activity, levels in serum, and in vivo activity, there are a number of complicating factors. The in vitro activities of macrolides are highly $\mathrm{pH}$ dependent, with activity decreasing with decreasing $\mathrm{pH}$. We have observed this relationship as well with $M$. leprae (unpublished observations) but have chosen to use a medium $\mathrm{pH}$ of approximately 6.5 , since this results in superior maintenance of bacillary metabolic activity than does a neutral $\mathrm{pH}$ (9). Therefore, the MIC in vitro may be considerably lower than that observed in this study. In a comparative study of the in vitro activities of macrolides against gram-positive cocci, sensitivities to medium acidification were, in ascending order, clarithromycin and erythromycin, roxithromycin, and azithromycin (2). In addition, macrolides concentrate intracellularly $(12,16)$, and therefore levels in serum are not necessarily indicative of concentrations in tissue (26). This fact may be of particular significance with $M$. leprae, which is considered an obligate intracellular organism.

Although it is conceivable that macrolide-resistant strains of $M$. leprae exist in nature, macrolides possess a number of properties which suggest potential for use in multidrug treatment of leprosy. They are relatively inexpensive, do not require refrigeration, are orally active, have low host toxicity and proven activity against a variety of intracellular pathogens $(2,4,5,7,24)$ but a restricted overall spectrum of activity, concentrate intracellularly to a greater extent than do any class of antimicrobial agents $(12,16)$, and act at a site (ribosome) not currently exploited in leprosy chemotherapy. In phase II and III clinical trials involving over 1,500 patients, clarithromycin administered at 250 to $500 \mathrm{mg}$ twice daily has been well tolerated (Murray Lumpkin, Abbott Laboratories, personal communication). On the basis of these properties and the results of this study, a clinical trial of clarithromycin in leprosy is warranted.

\section{ACKNOWLEDGMENTS}

We thank Noriko Matsunaga, Vernon Richard, and Ken White for excellent technical assistance.

This study was supported in part by Public Health Service grant R-22-AI22492 from the National Institute of Allergy and Infectious Diseases.

\section{LITERATURE CITED}

1. Arroyo, J., and G. Medoff. 1977. Mycobacterium chelonei infection: successful treatment based on a radiometric susceptibility test. Antimicrob. Agents Chemother. 11:763-764.

2. Barry, A. L., R. N. Jones, and C. Thornsberry. 1988. In vitro activities of azithromycin (CP 62,993), clarithromycin (A-56268; TE-031), erythromycin, roxithromycin, and clindamycin. Antimicrob. Agents Chemother. 32:752-754.

3. Berlin, O. G. W., L. S. Young, S. A. Floyd-Reising, and D. A. Bruckner. 1987. Comparative in vitro activity of the new macrolide A-56268 against mycobacteria. Eur. J. Clin. Microbiol. 6: 486-487.

4. Chang, H. R., and J.-C. F. Pechere. 1987. Effect of roxithromycin on acute toxoplasmosis in mice. Antimicrob. Agents Chemother. 31:1147-1149.

5. Chang, H. R., and J.-C. F. Pechère. 1988. In vitro effects of four macrolides (roxithromycin, spiramycin, azithromycin [CP62,993], and A-56268) on Toxoplasma gondii. Antimicrob. Agents Chemother. 32:524-529.

6. Chantot, J. F., A. Bryskier, and J. C. Gasc. 1986. Antibacterial activity of roxithromycin: a laboratory evaluation. J. Antibiot. 39:660-668.

7. Fernandes, P. B., R. Bailer, R. Swanson, C. W. Hanson, E. McDonald, N. Ramer, D. Hardy, N. Shipkowitz, R. R. Bower, and E. Gade. 1986. In vitro and in vivo evaluation of A-56268 (TE-031), a new macrolide. Antimicrob. Agents Chemother. 30: 865-873.

8. Franzblau, S. G. 1988. Oxidation of palmitic acid by Mycobacterium leprae in an axenic medium. J. Clin. Microbiol. 26:18-21.

9. Franzblau, S. G., and E. B. Harris. 1988. Biophysical optima for metabolism of Mycobacterium leprae. J. Clin. Microbiol. 26: 1124-1129.

10. Franzblau, S. G., E. B. Harris, and R. C. Hastings. 1987. Axenic incorporation of $\left[\mathrm{U}^{114} \mathrm{C}\right]$ palmitic acid into the phenolic glycolipid-I of Mycobacterium leprae. FEMS Microbiol. Lett. 48:407411.

11. Franzblau, S. G., and R. C. Hastings. 1987. Rapid in vitro metabolic screen for antileprosy compounds. Antimicrob. Agents Chemother. 31:780-783.

12. Gerding, D. N., L. R. Peterson, C. E. Hughes, and D. M. Bamberger. 1986. Extravascular antimicrobial distribution in man, p. 938-994. In V. Lorian (ed.), Antibiotics in laboratory medicine. The Willimas \& Wilkins Co., Baltimore.

13. Hastings, R. C., and S. G. Franzblau. 1988. Chemotherapy of 
leprosy. Annu. Rev. Pharmacol. Toxicol. 28:231-245.

14. Hastings, R. C., T. P. Gillis, J. L. Krahenbuhl, and S. G. Franzblau. 1988. Leprosy. Clin. Microbiol. Rev. 1:330-348.

15. Ji, B. 1985. Drug resistance in leprosy-a review. Lepr. Rev. 56:265-278.

16. Martin, J. R., P. Johnson, and M. F. Miller. 1985. Uptake, accumulation, and egress of erythromycin by tissue culture cells of human origin. Antimicrob. Agents Chemother. 27:314-319.

17. Mittal, A., M. Sathish, P. S. Seshadri, and I. Nath. 1983. Rapid, radiolabeled-microculture method that uses macrophages for in vitro evaluation of Mycobacterium leprae viability and drug susceptibility. J. Clin. Microbiol. 17:704-707.

18. Molaui, A., and L. Weinstein. 1971. In vitro activity of erythromycin against atypical mycobacteria. J. Infect. Dis. 123:216219.

19. Ramasesh, N., R. C. Hastings, and J. Krahenbuhl. 1987. Metabolism of Mycobacterium leprae in macrophages. Infect. Immun. 55:1203-1206.

20. Shepard, C. C. 1967. A kinetic method for the study of activity of drugs against Mycobacterium leprae in mice. Int. J. Lepr. 35: $429-435$.
21. Shepard, C. C., and D. H. McRae. 1968. A method for counting acid-fast bacteria. Int. J. Lepr. 36:78-82.

22. Shepard, C. C., R. M. Van Landingham, L. L. Walker, and R. C. Good. 1987. Activity of selected beta-lactam antibiotics against Mycobacterium leprae. Int. J. Lepr. 55:322-327.

23. Sibley, L. D., S. G. Franzblau, and J. L. Krahenbuhl. 1987. Intracellular fate of Mycobacterium leprae in normal and activated macrophages. Infect. Immun. 55:680-685.

24. Walsh, M., E. W. Kappus, and T. C. Quinn. 1987. In vitro evaluation of CP-62,933, erythromycin, clindamycin, and tetracycline against Chlamydia trachomatis. Antimicrob. Agents Chemother. 31:811-812.

25. Wheeler, P. R. 1988. Measurement of hypoxanthine incorporation in purified suspensions of Mycobacterium leprae: a suitable method to screen for anti-leprosy agents in vitro. J. Med. Microbiol. 25:167-174.

26. Wollmer, P., N. B. Pride, C. G. Rhodes, A. Sanders, V. W. Pike, A. J. Palmer, D. J. Silvester, and R. H. Liss. 1982. Measurement of pulmonary erythromycin concentration in patients with lobar pneumonia by means of position tomography. Lancet ii:13611364. 